\section{Practical Mathematics in Nuclear Medicine Technology}

\section{P. Wells}

Reston, VA: Society of Nuclear Medicine and Molecular Imaging, 2011, 331 pages, $\$ 85$

The softback, spiral-bound workbook Practical Mathematics in Nuclear Medicine Technology is a great tool for nuclear medicine technology students as well as a wonderful resource for the seasoned technologist. This second edition offers minor changes eliminating items that are no longer routinely used in nuclear medicine technology, such as the conversion of temperatures. The workbook is designed to ensure competency in basic math skills and to build on them with practical applications in nuclear medicine technology. The workbook also provides relevant regulations and background information that connect the mathematics to the everyday workings of a nuclear medicine technology department.

The 6 chapters include multiple sections. Each section is formatted similarly and isolates a specific process or procedural component, beginning with a "Principles" component, providing the background necessary to enhance understanding of the process. The next component is a shadow box that details the methods used to solve the problems. This step-by-step instruction is followed by one or more examples. The author then provides numerous practice problems for the user to complete. Some sections also include additional, more complex application problems that require the user to further manipulate the math involved. The sections conclude with a list of references. Notes from the author are provided throughout the book. These notes are items the author has found useful for understanding the material or for helping to prevent common mistakes. Specific regulations from the Nuclear Regulatory Commission or the Department of Transportation are included in those sections to which they pertain.

Three appendices accompany the book. The first is a list of decay charts for 20 common isotopes that are used in the practice problems. The second is a $\chi^{2}$ table. The third, and most significant, is solutions to all the practice problems. Most notable is that many of the solutions include how to set up the problem and do not simply provide an answer. This helps in identifying where an error was made.

Chapters 1 through 3 provide foundational mathematic skills that are applied in later chapters. These chapters do make every attempt to connect the mathematic skill to nuclear medicine technology concepts early on. Chapter 1 begins with a refresher on significant figures and basic mathematic operations. The chapter progresses through more complex mathematic operations such as exponents, proportions, and logs. These skills are then applied to conversions. Chapter 2 focuses on statistics used in nuclear medicine technology. The chapter does an excellent job of reviewing descriptive statistics and how to determine the error and SD of a series of values. The chapter also covers the nuances of counting rates. These foundations are applied to develop an understanding of the error and SD of single values and counting rates. Chapter 3 covers aspects of radiation protection. Although the shortest of the workbook, this chapter offers calculations that are germane to nuclear medicine technology and allow technologists to quantify exposure as it relates to time, distance, and shielding - a topic that is relevant for aspiring technologists and working technologists alike. Often students come from widely varying backgrounds and with widely varying skill sets. As an educator, I have found these first 3 chapters to be a barometer for student instructional needs and to help build the confidence of those students who have an aversion to mathematic calculations.

Calculations related to instrumentation, radiopharmacy, and clinical procedures are in chapters 4 through 6 . Chapter 4 uses previous concepts of error and $\chi^{2}$ to calibrate instrumentation, calculate acceptable ranges, and determine the accuracy and constancy of instrumentation. Chapter 5 addresses calculations used in the radiopharmacy to constitute kits, draw doses, perform quality control, and calculate pediatric doses. Mathematics associated with quantifying the outcomes of clinical procedures such as thyroid uptake, gallbladder ejection fraction, or whole blood volumes can be found in chapter 6 .

These last 3 chapters impart a foundation on calculations technologists use daily, providing an excellent, step-by-step learning tool to understand and perform those tasks. In addition, I recommend this workbook as a reference tool for any nuclear medicine technology department. Each year, I have the privilege of reviewing these calculations with a new cohort of students; however, many technologists have come to rely on the automation of these calculations in their daily routine. Computers have been extremely useful in converting wipe tests from counts per minute to disintegrations per minute or automatically retrieving a thyroid uptake percentage. What happens when those computers are not available? This workbook is a resource that is easily referenced and user-friendly.

This workbook is a must-have for aspiring technologists and an excellent resource for veteran technologists. In a world of automation, technologists still need to understand the essential mathematics within the profession. The author offers this profound thought in her preface: "Many of the mathematic computations technologists performed 10, 20, and 30 years ago are now being done for us. But the need to understand the mathematics behind the numbers we are handed is still paramount. Without this understanding we are mere technicians, rather than the expert technologists we strive to be."

\author{
Robert J. Loch, MBA, CNMT \\ Mercy College of Health Sciences \\ 928 Sixth Ave. \\ Des Moines, IA 50309 \\ E-mail: rloch@mercydesmoines.org
}

Published online Aug. 14, 2012.

DOI: 10.2967/jnmt.112.108639 